\title{
Skull base reconstruction after anterior subcranial tumor resection
}

\author{
Dan M. Fliss, M.D., Ziv Gil, M.D., Ph.D., Sergey SpeKtor, M.D., \\ Leonor Leider-Trejo, M.D., AVraham Abergel, M.D., AVi Khafif, M.D., \\ Aharon Amir, M.D., Eyal Gur, M.D., And Jacob T. Cohen, M.D.
}

Skull Base Surgery Unit, and Departments of Otolaryngology-Head and Neck Surgery, Neurosurgery, and Plastic and Reconstruction Surgery, Institute of Pathology, Tel-Aviv Sourasky Medical Center, Sackler Faculty of Medicine, Tel-Aviv University, Tel-Aviv, Israel

\begin{abstract}
Object. The goal of this study was to demonstrate the efficacy of a simple and reliable technique for anterior skull base and craniofacial reconstruction in patients who have undergone excision of tumors via the subcranial approach.

Methods. There were 63 patients who had undergone 71 anterior skull base resections of tumors via the aforementioned approach. Twenty-nine cases (41\%) involved malignant tumors and $42(59 \%)$ involved benign tumors. Reconstruction of the anterior skull base was performed by a single team who used double-layer fascial graft. Limited dural defects were reconstructed using the temporalis fascia, whereas large anterior skull base defects were reconstructed using a fascia lata sheath. Reconstruction was achieved without the support of bone graft or titanium mesh and without pericranial, galeal, or free flaps. Pericranial flap wrapping of the frontonasoorbital segment was performed to prevent osteoradionecrosis if postoperative radiotherapy was planned. The incidence of cerebrospinal fluid (CSF) leakage, intracranial infection, and tension pneumocephalus was 5.6\%. Histopathological examination of fascia lata grafts obtained in patients who had undergone a second procedure demonstrated integration of vascularized fibrous tissue to the graft, as well as local proliferation of a newly formed vascular layer embedding the fascial sheath.

Conclusions. The use of a double-layer fascial graft alone was adequate for prevention of CSF leakage, meningitis, tension pneumocephalus, and brain herniation. The double-layer fascial flap provided a simple and reliable means for anterior skull base reconstruction after en bloc resection of both malignant and benign tumors.
\end{abstract}

KEY WORDS • fascia lata • temporalis fascia $\bullet$ subcranial approach $\bullet$ skull base $\bullet$
reconstruction $\bullet$ malignant tumor

The technical development of anterior skull base surgery has had a major impact on the long-term survival and quality of life in patients with lesions involving the anterior cranial fossa. ${ }^{3,8,14,15,26,28}$ The concept of a subcranial approach to the entire anterior skull base in cases of traumatic injuries was first introduced by Raveh. ${ }^{21}$ This approach was later adopted by the same author as an alternative to the traditional transfacial-transcranial skull base approach for resection of tumors involving that anatomical region. ${ }^{11,23,24}$ Reconstruction of skull base and craniofacial defects is essential after tumor excision to: 1) form a watertight dural seal; 2) provide a barrier between the contaminated nasosinusoidal space and the sterile subdural compartment; 3) prevent airflow into the intracranial space; 4) maintain a functional sinonasal syste; and 5) provide a good cosmesis.

A variety of approaches have been developed to accom-

Abbreviations used in this paper: CSF = cerebrospinal fluid; $\mathrm{HA}=$ hydroxyapatite; $\mathrm{MR}=$ magnetic resonance. plish these goals. A split calvarial bone graft, HA paste, or titanium mesh may be used for osseous cranial reconstruction. Autologous flaps (pericranial flap, galeal flap), temporalis fascia or fascia lata grafts, free flaps (rectus abdominis flap, radial forearm flap, latissimus dorsi flap), and artificial substitutions are often used for reconstruction of the skull base. Unfortunately, these materials bear significant disadvantages. Local flaps are often insufficient in size for reliable restoration of extensive anterior skull base defects..$^{19}$ The microvascular technique for free flap reconstruction requires a highly specialized team, is more costly, and carries significant morbidity. ${ }^{5}$ Synthetic substitutions of dura and bone can induce chronic inflammation, carry a high risk of infection, and are inferior to biological sources in terms of strength and quality of the seal obtained..$^{29}$ Autografts, such as fascia lata and temporalis fascia, had been used in the past for skull base reconstruction, but they were usually covered with a vascularized flap (for example, free muscular flaps, pericranial, or galeal flaps), assuming that an overlying vascular tissue was essential to preserve long-term viability of the fascial graft. ${ }^{13}$ 
The failure to achieve an adequate reconstruction is associated with significant complications, such as CSF leakage, meningitis, brain herniation, and tension pneumocephalus. ${ }^{2}$ Although the subcranial approach has now become an established procedure for management of anterior skull base tumors, ${ }^{10,21-23}$ surgeons commonly use combinations of methods to accomplish satisfactory anterior skull base reconstruction. Thus, there is no single gold standard technique that is both simple and reliable for reducing the morbidity and mortality associated with anterior cranial base operations.

We present a large series of patients in whom extirpation of tumors via the subcranial approach was followed by reconstruction of the anterior skull base defect with a double-layer fascial flap. All operations were undertaken by one team who performed a single reconstruction method. We describe the healing process of the doublelayer free fascial grafts and provide evidence to show that long-term viability of the reconstruction can be achieved without requiring an overlying vascularized flap. The technique is simple, reproducible, and reliable for anterior skull base reconstruction, and it is associated with low rates of intracranial complications.

\section{CLINICAL MATERIAL AND METHODS}

This retrospective study was based on a review of the hospital charts and the outpatient clinical and neuroimaging data obtained in 71 consecutive patients in whom surgery was performed between 1994 and 2001. All resection and reconstruction procedures were conducted via a subcranial approach to the anterior skull base.

\section{Patient Population}

Thirty-five male and 28 female patients age 2 to 81 years (mean 42 years) were enrolled in this study. Preoperatively they were all evaluated by a head and neck surgeon, a plastic surgeon, and a neurosurgeon. Neurodiagnostic imaging studies included axial and coronal computerized tomography and MR imaging. Neuroangiography was also performed when indicated.

Broad-spectrum antibiotic therapy consisting of a combination of cefuroxime and metronidazole was instituted perioperatively. After induction of anesthesia, all patients underwent surgery in the supine position and without shaving of the hair at the surgical site. No tracheostomy was performed. A lumbar spine catheter was inserted for a period of 3 to 5 days for CSF drainage to facilitate frontal lobe retraction and to reduce the risk of postoperative CSF leakage. The mean hospitalization period was 13 days.

\section{Surgical Technique}

The surgical technique involved in the subcranial approach has been described in detail elsewhere. ${ }^{21-24}$ Briefly, after induction of anesthesia, the skin is incised above the hairline and a bicoronial flap is created in a supraperiosteal plane. A flap is elevated anteriorly beyond the supraorbital ridges and laterally superficial to the temporalis fascia. The pericranial flap is elevated up to the periorbits, and the supraorbital nerves and vessels are carefully separated from the supraorbital notch. The lateral and medial walls of the orbits are then exposed, and the ante- rior ethmoidal arteries are clipped or ligated. The pericranium is elevated above the nasal bones, and the flap is rotated forward and held over the face throughout the rest of the procedure. Titanium micro- or miniplates are applied to the frontal bones and removed before the osteotomies to ensure the exact repositioning of the osseous segments at the end of the operation. An osteotomy of the anterior or the anterior and posterior frontal sinus walls, together with the nasal bone frame, part of the medial wall of the orbit, and a segment of the superoposterior nasal septum, is then performed. For a Type A osteotomy, the anterior frontal sinus wall as well as the nasal frame are osteotomized and removed in one block. If a Type B osteotomy is planned, burr holes are made and the posterior frontal sinus wall is resected after the dura has been detached from the frontal, orbital, and ethmoidal roofs. A portion of the distal nasal bone is preserved to support the nasal valve. In cases in which a tumor invades laterally, the osteotomy lines can be extended to include segments of the orbital roofs. After the frontonasoorbital bone segment is osteotomized, it is stored in saline until the reconstruction procedure. A bilateral ethmoidectomy and a sphenoidotomy are then performed; this enables the exposure and assessment of the tumor in its circumference. The tumor is extirpated at this stage, and the dura or brain parenchyma is also resected when involved by tumor. Frozen sections are taken intraoperatively to ensure tumor-free margins. One or both sides of the cribriform plate and olfactory filaments are preserved whenever possible.

\section{Reconstruction Procedure}

The reconstruction technique initially described by Raveh, et al., ${ }^{23}$ is designed according to the size of the cranial defect, based on radiological and intraoperative calculations. Primary closure of the dura is performed whenever possible. A graft of temporalis fascia is used if the defect is limited. In cases of extensive skull base defects, a second surgical team simultaneously harvests a large $(20 \times$ $10-\mathrm{cm}$ ) fascia lata sheath. The size of the fascia used for reconstruction is tailored to the dimensions of the dural and skull base defects. The fascia is tacked under the edges of the dura and carefully sutured in place (Fig. 1). The repaired dural defect is then covered with a second layer of fascia applied against the entire under surface of the ethmoidal roof, the sella, and the sphenoidal area. Fibrin glue is used to provide additional protection against CSF leakage. We initially placed stents in the subcranial area; we used polyethylene tubes that were externalized in the nasal lumen, and the tubes were left in place for 6 months. In the last 10 patients in this series, however, we cranialized the frontal area. A centripetal compression method was used to reduce the telecanthus. In this method, two threads are guided through the medial canthal ligament and driven underneath the frontonasoorbital segment. The threads are tightened and fixed to the contralateral frontal plates to enable medial compression and alignment, thereby avoiding the telecanthus altogether. Vaseline-coated gauze is applied to the reconstructed skull base to provide additional support against dural pulsation. After removing all the mucosa from its undersurface, the earlier osteotomized segment is repositioned in its original anatomical place and fixed using prebent titanium plates. 


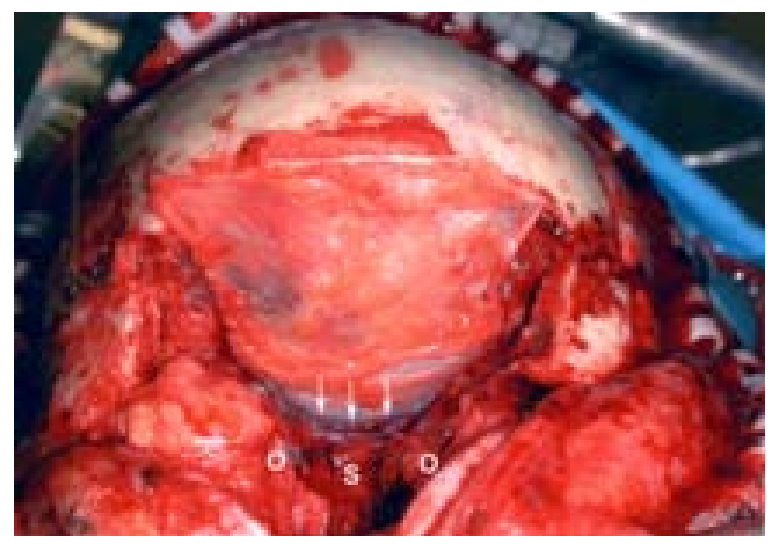

Fig. 1. Intraoperative photograph of the reconstruction area after tumor removal. A generous sheath of fascia lata has been applied to the skull base defect. The first layer is sutured to the dura at the margins of the defect (arrows) and reinforced using fibrin glue. $\mathrm{O}=$ orbits; $\mathrm{S}=$ posterior sphenoid sinus wall.

When the tumor involves the nasal bone or other frontoorbital segments, a split calvarial bone graft or posterior frontal sinus wall is used. A bone graft can also be used for posterior nasal support if the nasal septum has been resected. Reconstruction of the medial orbital walls is performed only in cases in which the total removal of this segment is necessary or if the periorbit is resected. In such cases, we use split calvarial bone grafts or three-dimensional titanium mesh covered by pericranium. We use HA paste (BoneSource; Leibinger, Dallas, TX) for small- or medium-sized defects of the calvarium following removal of the outer table (performed for grafting purposes).

In cases of eye globe exenteration, we use a temporalis muscle flap to cover the orbital socket: the anterior skull base is reconstructed using our standard double-layer fascial graft. A pericranial flap had been used in addition to a double-layer fascia lata only in one case in this series to cover a large anterolateral defect. We used no galeal or other regional flaps in this series. In patients undergoing extirpation of malignant tumors and planned adjuvant radiotherapy, we wrap the frontonasoorbital segment with a pericranial flap to prevent osteoradionecrosis (Fig. 2). A pericranial flap is also used in cases requiring reconstruction of the medial orbital wall, to cover the bone-graft segments or the titanium mesh.

Postoperatively, the patients are immediately transferred to the critical care unit for 24 hours. The lumbar drain is removed 3 to 5 days after the operation and the nasal packing is removed 8 days postoperatively. All patients were followed on a regular basis for a period of 3 to 50 months after discharge (mean follow-up period 26 months).

\section{Histopathological Examination}

We investigated the healing process of fascia lata grafts in three patients who had undergone a second surgery. Fragments of fascia lata that had previously been used for skull base reconstruction and in which there was no tumor involvement were excised and studied histologically. The fascia lata segment was fixed in $10 \%$ buffered formalin. Each specimen was embedded in paraffin and serially

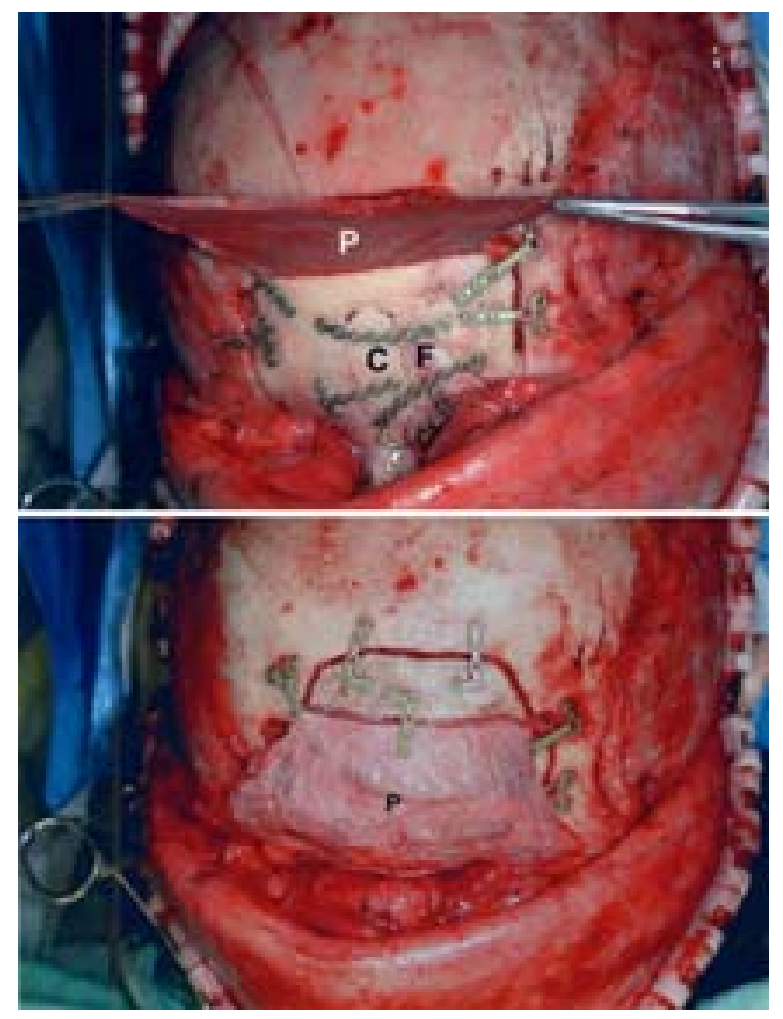

Fig. 2. Photographs demonstrating the reconstruction of the frontonasoorbital segment and coating with a pericranial flap. Upper: In cases of tumor invasion, bone reconstruction of the frontonasoorbital segment is performed using outer-table calvarial bone or posterior frontal sinus wall grafts. Lower: If adjuvant radiotherapy is planned, the whole segment is coated with a pericranial flap. This vascularized tissue is guided underneath the osseous segment to cover the intranasal surface and than externalized over the entire frontal area. $\mathrm{C}=$ outer-table calvarial bone graft; $\mathrm{F}=$ posterior frontal sinus wall graft; $\mathrm{P}=$ pericranial flap.

sliced ( $4 \mu \mathrm{m}$ in thickness). The microsections were stained with hematoxylin and eosin. The degree of vascular proliferation and fibrotic reaction was evaluated by a senior pathologist.

\section{RESULTS}

Surgery-related outcomes in 63 patients who underwent 71 procedures involving anterior skull base tumors were analyzed. Malignant tumors were present in 29 cases $(41 \%)$ and benign tumors were present in 42 cases $(59 \%)$. There was no significant difference in age or sex between the two groups. The most common malignant tumor was squamous cell carcinoma (eight cases) and the most common benign lesion was meningioma (12 cases). Table 1 provides a summary of all underlying pathological lesions in our patients.

Twenty-nine patients $(41 \%)$ had undergone at least one previous operation. Twenty-seven patients (38\%) underwent perioperative radiotherapy (11 patients pre- and 16 patients postoperative radiotherapy), of whom 15 (21\%) had also undergone at least one previous operation. The subcranial approach was used as a single procedure in 57 cases. It was combined with a midfacial degloving proce- 
D. M. Fliss, et al.

TABLE 1

Histopathology of anterior skull base lesions*

\begin{tabular}{|c|c|c|c|c|c|}
\hline \multirow[b]{2}{*}{ Lesion } & \multirow[b]{2}{*}{ No. } & \multicolumn{4}{|c|}{ Patient Outcome (\%) } \\
\hline & & $\mathrm{A} \& \mathrm{~W}$ & AWD & DOD & DOC \\
\hline meningioma & 12 & 11 & 1 & & \\
\hline mucocele & 10 & 9 & & & 1 \\
\hline osteoma & 4 & 4 & & & \\
\hline inverted papilloma & 4 & 3 & & & 1 \\
\hline neurofibromatosis Type 1 & 2 & 2 & & & \\
\hline arteriovenous malformation & 2 & 2 & & & \\
\hline meningoencephalocele & 2 & 2 & & & \\
\hline pituitary adenoma & 1 & 1 & & & \\
\hline hemangioma & 1 & 1 & & & \\
\hline chordoma & 1 & 1 & & & \\
\hline epidermoid cyst & 1 & 1 & & & \\
\hline fibrous dysplasia & 1 & 1 & & & \\
\hline angiofibroma & 1 & 1 & & & \\
\hline squamous cell carcinoma & 8 & 6 & & 1 & 1 \\
\hline esthesioneuroblastoma & 7 & 3 & 3 & 1 & \\
\hline adenocarcinoma & 3 & 1 & 2 & & \\
\hline plasmocytoma & 2 & 2 & & & \\
\hline melanoma & 2 & 1 & 1 & & \\
\hline sarcoma & 2 & 1 & 1 & & \\
\hline hemangiopericytoma & 2 & 1 & & & 1 \\
\hline adenoid cystic carcinoma & 1 & & 1 & & \\
\hline malignant schwannoma & 1 & & 1 & & \\
\hline lymphoma & 1 & & & & 1 \\
\hline total & 71 & $54(76)$ & $10(14)$ & $2(3)$ & $5(7)$ \\
\hline
\end{tabular}

$* \mathrm{AWD}=$ alive with disease; $\mathrm{A} \& \mathrm{~W}=$ alive and $\mathrm{well} ; \mathrm{DOC}=$ died of other causes; $\mathrm{DOD}=$ died of disease

dure $^{9}$ in six cases, with a pterional approach ${ }^{30}$ in six patients, and with a transorbital approach in two cases.

The principal skull base reconstruction procedure was performed using a double-layer fascia lata (62 cases). In 50 of these cases, the tumor extended to the dura mater, which was also removed and reconstructed using a fascia lata graft. The remaining cases included seven in which the dura was reconstructed using temporalis fascia, and two in which no dural reconstruction was required.

We used the temporalis muscle flap in five cases of orbital exenteration and performed the same double-layer fascial flap procedure for anterior skull base reconstruction.

A craniofacial reconstruction was required if the tumor resection produced a significant osseous defect of the orbital walls, nasal bone, or anterior frontal sinus wall. This was achieved by applying a split calvarial bone graft or posterior sinus wall (eight and five cases, respectively). A titanium mesh covered with a pericranial flap was used for reconstruction of the medial orbital walls and to cover extensive calvarial defects in four cases. We used HA paste to fill defects in the calvarium after harvesting the outer table for reconstruction in six other cases.

The overall complication rate was $43 \%$; however, the incidence of CSF leak, intracranial infection, and tension pneumocephalus was $5.6 \%$. One patient who underwent surgery for a pituitary adenoma and who suffered from meningitis died 42 days after surgery. The overall postoperative complications associated with skull base and cranial reconstruction are listed in Table 2. Fistula-related osteoradionecrosis was found in five patients, four of
TABLE 2

Complications associated with skull base reconstruction procedures

\begin{tabular}{ll}
\hline \hline \multicolumn{1}{c}{ Complication } & No. (\%) \\
\hline meningitis & $2(2.8)$ \\
CSF leak & $1(1.4)$ \\
tension pneumocephalus & $1(1.4)$ \\
intracranial hematoma & $2(2.8)$ \\
osteoradionecrosis \& fistula & $5(7)$ \\
wound infection & $5(7)$ \\
mucocele & $2(2.8)$ \\
telecanthus & $5(7)$ \\
ptosis & $2(2.8)$ \\
epiphora & $5(7)$ \\
facial nerve paralysis & $1(1.4)$ \\
total & $31(43.6)$ \\
\hline
\end{tabular}

whom had undergone perioperative radiotherapy. Because of the risk for osteoradionecrosis of the frontonasoorbital segment in patients who undergo perioperative radiotherapy, we developed a new method for skull base reconstruction. In these cases we used the pericranial flap for wrapping the frontonasoorbital segment (Fig. 2). There were no cases of bone flap necrosis in the three patients who underwent this procedure. In two cases of wound dehiscence, that was associated with temporalis muscle transfer following orbital exenteration, the patients were successfully treated by applying local flaps several days postoperatively. The complication rates in cases of benign and malignant tumors were similar.

The oncological outcome in this series is shown in Table 1. Two patients died of their diseases, five died of unrelated causes, 54 are without evidence of disease, and 10 patients are alive with disease.

We investigated the healing process of fascia lata grafts in three patients who had undergone a second surgery. Fragments of the fascia lata that were previously used for skull base reconstruction and were not involved with the tumor were excised and histopathologically evaluated. Microscopic examination showed that the entire graft was composed of viable dense fibrocollagenous tissue. In large areas the bipolar, wavy shaped nuclei of the fibroblasts were embedded in a collagenous stroma. The fibroblastic reaction was accompanied by proliferation of neovascular channels lined by plumped endothelial cells. The histological findings demonstrated an almost complete fibrous replacement of the fascia lata allograft (Fig. 3).

\section{DISCUSSION}

Various surgical techniques for extirpation of anterior skull base tumors have been established over the past 30 years. ${ }^{7,16,23,25}$ Tumors that are located in this anatomical area may invade both soft and hard tissues of the skull base. In such cases, an en bloc tumor resection may create extensive skull base defects and produce a free conduit between the paranasal sinuses and the intracranial space. Following tumor resection, cranial base defects require reconstruction to create a secure barrier between these two compartments. Failure of the reconstruction carries potential life-threatening complications (for example, CSF 


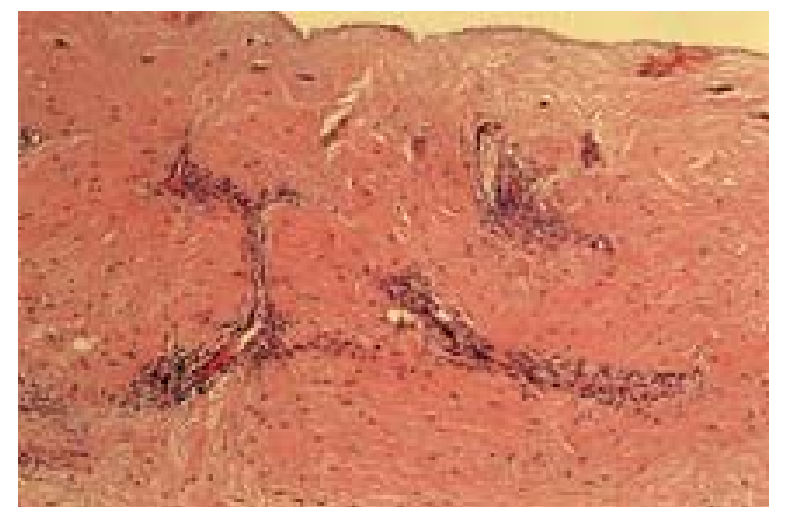

Fig. 3. Photomicrograph of integrated fascia lata graft, showing fibroblasts embedded in a dense collagenous stroma. Note the presence of neovascularized channels lined by endothelial cells. The graft has been replaced with viable dense fibrocollagenous tissue. $(\mathrm{H} \& \mathrm{E}$, original magnification $\times 20)$.

leakage and meningitis) that may delay the initiation of adjuvant therapy.

Reconstruction of the anterior skull base is technically challenging and may be further complicated by several factors. First, there is a paucity of local tissue that is available for transfer into the defect. Second, previous radiotherapy significantly reduces tissue perfusion that delays normal wound healing. Finally, many of these patients have undergone multiple surgeries prior to the index operation, thus increasing its complexity and, secondary to scar tissue formation, decreasing tissue perfusion.

Regional flaps have been shown to be a reasonable option for dural reconstruction. ${ }^{19}$ This technique is often sufficient for forming a barrier to separate the dura from the nasopharynx. The pericranial and galeal flaps are the most common regional flaps chosen for this purpose. They are readily available, easily harvested, and can be used to repair various dural defects. Galeal flaps may not always be available, however, particularly after radiotherapy or previous operations. They can also cause functional and cosmetic complications, including sensorimotor loss and regional alopecia. ${ }^{27}$ Furthermore, the use of galeal or pericranial flaps may be inadequate for covering defects of the frontal bone (for example, burr holes and craniotomy bone cuts), which are readily visible through the thinned skin. Because loss of galea and frontalis muscle obtained from the undersurface of the frontal scalp results in a very thin and poorly vascularized flap of skin for covering the bone flap, its postirradiation use is controversial.

In recent years, progress in various microvascular and surgical techniques has enabled the development and implementation of free-tissue transfer. Free flaps may be used for massive skull base defects, with excellent surgery-related results and low complication rates. ${ }^{4,19}$ The rectus abdominis is the most commonly used free flap in this anatomical area, and the radial forearm and latissimus dorsi flaps are the second most common. Furthermore, free-tissue transfer promises flexibility in flap content and design, and it provides the opportunity to introduce a large quantity of well-vascularized tissue to the reconstructed area in a single-stage operation. Significant morbidity and mortality rates, however, have been documented in elderly patients who underwent free-flap reconstructions. ${ }^{5}$ Free-tissue transfer is a relatively complex surgical procedure, requiring especially advanced technical qualifications. Another drawback of this method is the bulk of the muscular free flap, which may mask local recurrence and make radiological follow up more difficult. ${ }^{17}$

In the current study, we used a double-layer fascial sheath as the standard material for anterior skull base reconstruction. In a previous report ${ }^{1}$ we described a simple technique for harvesting large fascia lata sheaths, which yielded a low complication rate and low donor-limb morbidity. The thin and low mass properties of the fascia lata enable the surgeon to cover large dural defects with a single fascial sheath. Furthermore, the flexibility of the fascia lata enables coating of extensive cranial defects, including parts of the orbit and paranasal sinuses. Large cranial base defects and prior surgery and radiotherapy (previously considered indications for free-flap reconstruction ${ }^{18}$ ) were managed using fascia alone. Free flaps, autologous fat, or skin grafts were not necessary for achieving a reliable skull base reconstruction that yielded excellent surgery-related outcome.

Several authors have contended that osseous reconstruction of the skull base is necessary to support the newly reconstructed skull base and to prevent herniation of the cranial content. ${ }^{6}$ Analysis of our current results, however, showed that reconstruction using a double-layer fascia does not require a rigid support of bone or of synthetic materials; no case of brain herniation occurred in our series of 71 procedures.

The overall duration of the reconstructive procedure was no longer than that required for a pericranial flap, ${ }^{28}$ and it takes considerably less time than that for free-tissue transfer procedures. ${ }^{20}$

Routine postoperative neuroimaging evaluation is required in all patients in whom malignant anterior skull base tumors have been excised. The relatively low bulk of the fascia lata assists in follow-up neuroimaging studies in patients in whom there is an increased risk of local recurrence (Fig. 4).

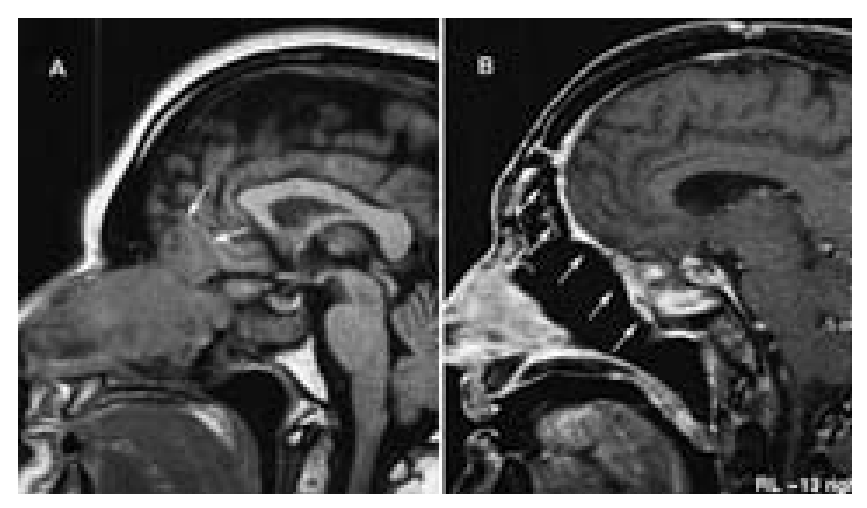

Fig. 4. Magnetic resonance images obtained in a patient with squamous cell carcinoma. A: Preoperative midsagittal MR image revealing nasal and intracranial extensions (arrows) of the tumor. B: Postoperative midsagittal MR image demonstrating area after removal of tumor and placement of layers of fascia lata (arrows). 
The use of fascial flaps does not require the utilization of skin grafts or muscle flaps facing the nasopharyngeal space. In our patients transnasal endoscopic examination several weeks postoperatively revealed that the exposed nasal surface of the flap had been completely covered by the neighboring sinonasal mucosa.

Wrapping of the pericranial flap of the frontonasoorbital segment was performed to prevent osteoradionecrosis if postoperative radiotherapy was planned. We speculate that such a procedure may provide an excellent supply of blood and nutrients to the osteotomized bone, from an overlying regional flap. Although the sample of cases in which we used this method is rather small (three patients), there were no cases of bone flap necrosis in this group. Further studies are required to determine whether this approach indeed lowers the risk of osteoradionecrosis.

Although the advantages of the extended subcranial approach have been outlined, there are still clinical situations in which, in our opinion, it should be either replaced by other classic techniques or coupled with complementary procedures. These situations include lesions involving the cavernous sinus or the carotid arteries and massive intradural or brain invasion. A pterional approach in addition to the subcranial approach may be performed in cases of tumors extending posterolaterally. If the tumor extends inferolaterally, an additional infratemporal fossa approach may be used. In cases in which tumors involve the anterior fossa skull base and the maxillary lateral walls or alveolar ridge, a combined subcranial-midfacial degloving approach may offer improved exposure and excellent cosmesis results. Nevertheless, in all these cases we use the standard double-layer fascia lata for reconstruction of the skull base.

In this work, we also studied the histopathological effect of the reconstructed fascia lata to investigate the healing process of the reconstructed area in patients who had undergone a second operation. Our histological examination of previously harvested human fascial flaps showed evidence of integration of a vascularized fibrous tissue into the fascial graft. The fascial flap was uniformly coated by fibrous tissue, and invasion of blood vessels was achieved without the presence of an overlying vascularized flap in all three fascial flaps that were examined. In an animal model, Tachibana, et al., ${ }^{29}$ demonstrated a tight connection between the fascial graft and the dura within 1 week after surgery, which is in agreement with our findings. They reported that the fascial graft had been completely replaced by durable fibrous tissue 2 weeks following the surgical repair, and they speculated that fibroblast growth factor- $\beta$ plays a significant role in the healing process of free fascial grafts. ${ }^{12,29}$ Thus, results obtained in our current work and those in the animal model confirm that excellent skull base reconstruction can be achieved without requiring blood supply from an overlying regional flap or free muscular flap. Moreover, the rapid healing process of the fascial reconstruction provides a robust physiological barrier between the nasopharynx and the intracranial space within days following surgery.

\section{CONCLUSIONS}

Reconstruction after resection procedures of anterior skull base tumors vary widely. In our experience the double-layer fascia technique promises a simple and versatile means of skull base reconstruction after en bloc resection of malignant and benign tumors. The incidences and severity of perioperative complications associated with the double-layer fascia lata technique is similar to or lower than those of other reconstructive techniques. Our findings also indicate that free fascial grafts survive due to local proliferation of a newly formed vascular layer embedded within the fascial sheath.

Future developments of biomedical materials will likely yield continuing improvement in current techniques for skull base reconstruction-there will be fewer complications and the promise of a better quality of life for patients.

\section{References}

1. Amir A, Gatot A, Zucker G, et al: Harvesting large fascia lata sheaths: a rational approach. Skull Base Surg 10:29-34, 2000

2. Boyle JO, Shah KC, Shah JP: Craniofacial resection for malignant neoplasms of the skull base: an overview. J Surg Oncol 69:275-284, 1998

3. Cheesman AD, Lund VJ, Howard DJ: Craniofacial resection for tumors of the nasal cavity and paranasal sinuses. Head Neck Surg 8:429-435, 1986

4. Clayman GL, DeMonte F, Jaffe DM, et al: Outcome and complications of extended cranial-base resection requiring microvascular free-tissue transfer. Arch Otolaryngol Head Neck Surg 121:1253-1257, 1995

5. Coleman JJ III: Microvascular approach to function and appearance of large orbital maxillary defects. Am J Surg 158: 337-341, 1989

6. Derome P: The transbasal approach to tumors invading the base of the skull, in Schmidek HH, Sweet WH (eds): Operative Neurosurgical Techniques, ed 2. New York: Grune \& Stratton, 1988, pp 619-634

7. Donald PJ: Complications in skull base surgery for malignancy. Laryngoscope 109:1959-1966, 1999

8. Donald PJ: Skull base surgery combined results of treatment of malignant disease. Skull Base Surg 2:76-79, 1992 (Reference unverified)

9. Fliss DM, Zucker G, Amir A, et al: The combined subcranial and midfacial degloving technique for tumor resection: report of three cases. J Oral Maxillofac Surg 58:106-110, 2000

10. Fliss DM, Zucker G, Amir A, et al: The subcranial approach for anterior skull base tumors. Oper Techn Otolaryngol Head Neck Surg 11:238-253, 2000

11. Fliss DM, Zucker G, Cohen A, et al: Early outcome and complications of the extended subcranial approach to the anterior skull base. Laryngoscope 109:153-160, 1999

12. Folkman J, Klagsbrun M: Angiogenic factors. Science 235: $442-447,1987$

13. Hasegawa M, Torii S, Fukuta K, et al: Reconstruction of the anterior cranial base with the galeal frontalis myofascial flap and the vascularized outer table calvarial bone graft. Neurosurgery 36:725-731, 1995

14. Irish JC, Gullane PJ, Gentili F, et al: Tumors of the skull base: outcome and survival analysis of 77 cases. Head Neck 16: 3-10, 1994

15. Jackson IT: Craniofacial osteotomies to facilitate resections of tumors of the skull base, in Wilkins RH, Rengachary SS (eds): Neurosurgery Updates I: Diagnosis, Operative Technique, and Neuro-Oncology. New York: McGraw-Hill, 1990, pp 227-290 (Reference unverified)

16. Kellman RM, Marentette L: The transglabellar/subcranial approach to the anterior skull base: a review of 72 cases. Arch Otolaryngol Head Neck Surg 127:687-690, 2001 


\section{Postresection skull base repair}

17. Kiyokawa K, Tai Y, Inoue Y, et al: Efficacy of temporal musculopericranial flap for reconstruction of the anterior base of the skull. Scand J Plast Reconstr Surg Hand Surg 34:43-53, 2000

18. McCutcheon IE, Blacklock JB, Weber RS, et al: Anterior transcranial (craniofacial) resection of tumors of the paranasal sinuses: surgical technique and results. Neurosurgery 38: 471-480, 1996

19. Neligan PC, Mulholland S, Irish J, et al: Flap selection in cranial base reconstruction. Plast Reconstr Surg 98:1159-1166, 1996

20. Nibu K, Sasaki T, Kawahara N, et al: Complications of craniofacial surgery for tumors involving the anterior cranial base. Neurosurgery 42:455-462, 1998

21. Raveh J: Gesichtsschadelverletzungen: Eigene Erfahrungen und modificationen. Aktuel Probl ORL 3:145-154, 1979 (Reference unverified)

22. Raveh J, Laedrach K, Lizuka T, et al: Subcranial extended anterior approach for skull base tumors: Surgical procedure and reconstruction, in Donald PJ (ed): Surgery of the Skull Base. New York: Lippincott-Raven, 1998, pp 239-261

23. Raveh J, Laedrach K, Speiser M, et al: The subcranial approach for fronto-orbital and anteroposterior skull-base tumors. Arch Otolaryngol Head Neck Surg 119:385-393, 1993

24. Raveh J, Turk JB, Ladrach K, et al: Extended anterior subcra- nial approach for skull base tumors: long-term results. J Neurosurg 82:1002-1010, 1995

25. Roux FX, Moussa R, Devaus B, et al: Subcranial fronto-orbitonasal approach for ethmoidal cancers surgical techniques and results. Surg Neurol 52:501-510, 1999

26. Sekhar LN, Nanda A, Sen CN, et al: The extended frontal approach to tumors of the anterior, middle, and posterior skull base. J Neurosurg 76:198-206, 1992

27. Snyderman CH, Janecka IP, Sekhar LN, et al: Anterior cranial base reconstruction: role of galeal and pericranial flaps. Laryngoscope 100:607-614, 1990

28. Sundaresan N, Shah JP: Craniofacial resection for anterior skull base tumors. Head Neck Surg 10:219-224, 1988

29. Tachibana E, Saito K, Fukuta K, et al: Evaluation of the healing process after dural reconstruction achieved using a free fascial graft. J Neurosurg 96:280-286, 2002

30. Zucker G, Nash M, Gatot A, et al: The combined subcranialpterional approach to the anterolateral skull base. Oper Techn Otolaryngol Head Neck Surg 11:286-293, 2000

Manuscript received March 19, 2002.

Accepted in final form April 22, 2002.

Address reprint requests to: Dan M. Fliss, M.D., Skull Base Unit, Tel-Aviv Sourasky Medical Center, 6 Weizmann Street, Tel-Aviv 64239, Israel. email: fliss@ tasmc.health.gov.il. 